Research Article

\title{
Evaluation of Antibacterial Activity of Essential Oils and Their Combination against Multidrug-Resistant Bacteria Isolated from Skin Ulcer
}

\author{
Eshetu Gadisa $\mathbb{D}^{1}$ and Hydar Usman $\mathbb{D}^{2}$ \\ ${ }^{1}$ Department of Medical Laboratory Science, MMHSC, KMU, Addis Ababa, Ethiopia \\ ${ }^{2}$ Department of Pharmacy, Health Science College, Madda Walabu University, Bale Robe, Ethiopia
}

Correspondence should be addressed to Eshetu Gadisa; gadisaeshetu@gmail.com

Received 5 October 2020; Revised 9 February 2021; Accepted 5 March 2021; Published 27 March 2021

Academic Editor: Giuseppe Comi

Copyright (c) 2021 Eshetu Gadisa and Hydar Usman. This is an open access article distributed under the Creative Commons Attribution License, which permits unrestricted use, distribution, and reproduction in any medium, provided the original work is properly cited.

\begin{abstract}
Background. Emerging of multidrug-resistant bacteria can compromise the effectiveness of antibiotics used to treat skin infections. Those bacteria imposed public health problems and questioning medical care in the $21^{\text {st }}$ century. In this circumstance, essential oils of medicinal plants origin are supreme sources of structural and functionally divergent compounds, which inhibited the growth of common wound colonizing MRSA and ESBL producing P. aeruginosa. The aim of this study was to evaluate the combined antibacterial activity of essential oils extracted from Rumex abyssinicus, Cucumis pustulatus, and Discopodium penninervium against multidrug-resistant (MDR) isolates of skin ulcers. Methods. Essential oils (EOs) were extracted from aerial parts of R. abyssinicus, C. pustulatus, and D. penninervium with steam distillation. A mixture of each oil $(1: 1)$ was adsorbed to a disc and placed on Mueller Hinton Agar. Then, minimum zone of inhibition and bactericidal concentration of EOs was measured after incubeted for $18-24$ hours at $37^{\circ} \mathrm{C}$. Their combined antibacterial effect was determined by the fractional inhibitory concentration index. Results. The antibacterial activity of mixed oil varied in their doses and bacteria species, of which a mixture of essential oil of $R$. abyssinicus and D. penninervium had inhibition zone ( $32 \mathrm{~mm})$; its MIC and MBC values range from $1-2 \mu \mathrm{l} / \mathrm{ml}$ against MRSA. It had an inhibition zone $(36 \mathrm{~mm})$, MIC value $4 \mu \mathrm{l} / \mathrm{ml}$, and $\mathrm{MBC}(8 \mu \mathrm{l} / \mathrm{ml})$ against ESBL producing $P$. aeruginosa, whereas combined effects of $R$. abyssinicus and C. pustulatus had MIC values ranging from $2-8 \mu \mathrm{l} / \mathrm{ml}$ for E. coli and K. pneumoniae and $2 \mu \mathrm{l} / \mathrm{ml}$ for MRSA. There was a strong synergistic effect between $R$. abyssinicus and D. penninervium and promising antibacterial effect more specifically on MRSA and P. aeruginosa. Conclusion. This in vitro study of the combined effect of EOs has significant antibacterial activity on wound colonizing bacteria and reduces delaying wound healing as that of modern drugs tested in parallel. Hence, further structural elucidation of active compounds helps us to properly design or synthesis of topical antibiotics for wound care.
\end{abstract}

\section{Background}

Modern humans have universally used medicinal plants for healing properly many ailments $[1,2]$. Evolving of the powerful analytical tools based upon proteomics, metabolomics, and genomics can aid to discover novel compounds from medicinal plants. The compounds of plant origin have divergent chemical structures and functionally relevant precursor molecules to discover antibiotics $[2,3]$. Besides this, widely ethnobotanical and ethnopharmacological studies of essential oils from plants contributed to finding various compounds, of which leptospermone, tricyclene, flavesone, myrcene, carvacrol, p-cymene, eugenol, $\gamma$-terpinene, phenylpropanoids, $\beta$-selinene, and calamenene are some important compounds inhibiting the growth and biofilm formation of pathogen bacteria and used as immunomodulatory compounds $[4,5]$. For instance, carvacrol caused collapse of the proton-motive force and depletion of the ATP pool, with consequent cell death [6]. Therefore, essential oils are 
applicable as precursors in the pharmaceutical industries for development of antibiotics $[7,8]$.

Nowadays, there is an increasing attention in exploring potential therapeutic bioactive to treat serious ailments caused by multidrug-resistant bacteria such as chronic wound $[3,4,8,9]$. WHO reported that there were more than 8.2 million people infected with wounds with or without infection. This caused \$28.1-96.8 billion lost to treat acute and chronic wounds. In this regard, the United States lost $\$ 25$ billion per year for healthcare expenditure for nonhealing ulcer [10]. Likewise, a study conducted in Europe showed that there were 1.5-2 million people agonized from wounds. Unless and otherwise invented for new novel treatment, it imposed serious economic impact and costs \$15-22 billion per year in coming five years. It has become major public health problem that caused psycho-social consequence on infected patients $[9,10]$. Emerging of MRSA, VRE, and ESBL producing $P$. aeruginosa that identified as common wound colonized MDR bacteria has been worsen medical-surgical care and other health services [9-11]. In such case, essential oils of medicinal plants origin are suitable candidates to develop topical ointments for wound care and beyond $[8,12]$.

Essential oils have many compounds that can aid wound healing. In this regard, essential oil able to immunomodulating potential to typically activate both humoral and cell-mediated immune response. Other compounds such as carvacrol have bactericidal property through inhibition of protein and nucleic acid synthesis of MRSA and ESBL producing Enterobacteriaceae $[5,12,13]$. Some of them prevent biofilm formation and multiplication, inhibitory effect on inflammatory edema formation, and leucocyte chemotaxis around the wound. Therefore, essential oils are improving the quality wound care, decrease morbidity, and mortality and overcoming nonhealing trajectory and low therapeutic response of chronic wound infection $[8,12,13]$.

Many studies showed that combined effect of essential oils had powerful antibacterial activity on wound colonizing pathogen multidrug-resistant bacteria $[3,12,13]$. The EOs of Cinnamomum verum and piperacillin mixture had synergistic activity against beta-lactamase TEM-1E. coli. This combination made wrecked the outer membrane or QS inhibition in bacteria cells [14]. Interestingly, a study conducted on Qingre Baidu mixture could inhibit the biofilm formation of $S$. aureus and $P$. aeruginosa. It reduces AI-2 level and upregulating expression of HIF- $1 \alpha$, HIF- $2 \alpha$, and HIF- $3 \alpha$, which increased the levels of VEGF, thereby promoting angiogenesis and wound healing in chronic and refractory wounds [15]. Another study showed that EOs of Pilgerodendron uviferum and Melaleuca alternifolia can inhibit efflux pump mechanism of the S. aureus NorA. These essential oils can inhibit efflux pump and characterize a potentially safe and affordable ingredients to develop skin friendly ointment to wound colonizing multidrug-resistant bacteria [16-20].

Currently, using combined conventional antibiotics and essential oils or essential oils themselves as wound care ointments are promising strategies to overcome multidrugresistant bacteria. MRSA and ESBL producing E. coli have resisted for amoxicillin, tetracycline, piperacillin, ofloxacin, and oxacillin [21-23]. Many studies showed that combined effect of Leptospermum scoparium and Tri-EDTA; Mentha piperita and Micromelum integerrimum and; cinnamon bark oil and cinnamaldehyde had synergistic antibacterial activity on MSSA, E. coli, MRSA, ESBL, and P. aeruginosa [24, 25]. From these viewpoints, we purposed to evaluate combined antibacterial activity of EOs extracted from R. abyssinicus, C. pustulatus, and D. penninervium against bacteria isolated from wounds. Essential oils had immunomodulatory compounds that serve as a baseline data to formulate and designing novel compounds and find out the scientific rationale for the combined effects of untapped medicinal plants used by different societies.

\section{Materials and Methods}

\subsection{Study Area and Design}

2.1.1. Study Area. This study was conducted in the Seweyna woreda, Bale Zone. This woreda is located $437 \mathrm{~km}$ away from Robe (zonal town) in the northern east direction and $750 \mathrm{~km}$ from the capital city, Addis Ababa. An elevation extends from $400-1850 \mathrm{~m}$ above sea level and is located at coordinates latitude $7^{\circ} 19^{\prime} 60.00^{\prime \prime} \mathrm{N}$ and longitude $41^{\circ} 19^{\prime} 60.00^{\prime \prime}$ E. Major rivers include the Mekenisa, Dare, Manduba, and Kurkura. According to the national land survey, Seweyna is covered by abundant pasture (46.3\%), arable or cultivable (24.4\%) and forest or heavy vegetation (24.1\%). This district is the remotest area with no infrastructure (transport, hospital, and power supplies). There has been one health center and residents depend on traditional knowledge of TMP to treat ailments such as skin diseases, diabetes, STI cancer, hypertension, and impotence.

2.1.2. Study Design and Period. An in vitro experimental study was carried out to evaluate the antibacterial effect of combined essential oils, $R$. abyssinicus, $C$. pustulatus, and $D$. penninervium, against MDR and their reference strains at KMU, Core Laboratory, from January to April, 2020.

2.2. Medicinal Plants Selection Criteria. Nowadays, pastoralist communities are deprived of modern medical care and depend on the traditional medicinal plants to treat many human and animals' ailments such as skin diseases, diabetes, hepatitis, and cancer. These medicinal plants were selected based on traditional knowledge of healers to treat bacterial infections such as eczema, gonorrhea, syphilis, pneumonia, scabies, and other skin infections commonly with R. abyssinicus, C. pustulatus, and D. penninervium.

2.3. Plant Collection and Extraction. Plant samples of $R$. abyssinicus, C. pustulatus, and D. penninervium were collected from sideways of Mekenisa and Kurkura rivers of Seweyna woreda. Each plant was euthanatized and deposited at the National Herbarium with Rumex abyssinicus voucher number (E-55/07), Cucumis pustulatus (E-45/07), and Discopodium penninervium (E-18/07) in the Department of 
Biology, Faculty of Natural and Computational Science, AAU. Well-grown aerial part of each plant was collected and extracted its essential oils with steam distillation using AMIO-37/04 model for 3-5 hours and stored in brown colored bottle vials at $4{ }^{\circ} \mathrm{C}$ as described by Eshetu [3].

\subsection{Culture Media and Multidrug Bacteria}

2.4.1. Culture Media. Nutrient agar, TSY broth, MacConkey, MHA, MHB, BA, mannitol salt agar, chocolate agar, and other reagents were used to grow bacteria collected from wounds.

2.4.2. Test Organisms. The reference bacterial species of E. coli (ATCC25922), K. pneumoniae (ATCC700603), E. faecalis (ATCC 29212) and S. aureus (ATCC 25923), $P$. aeruginosa (ATCC 27853), and their MDR strains were isolated from wounds of out-patients attending Menelik Hospital, Addis Ababa. Ciprofloxacin $(5 \mu \mathrm{g})$, gentamycin $(10 \mu \mathrm{g})$, cephalotaxine $(30 \mu \mathrm{g})$, cefotaxime $(5 \mu \mathrm{g})$, ceftazidime $(10 \mu \mathrm{g})$, cefoxitin $(30 \mu \mathrm{g})$, ceftriaxone $(30 \mu \mathrm{g})$, amikacin $(30 \mu \mathrm{g})$, cefuroxime $(5 \mu \mathrm{g})$, ceftriaxone $(30 \mu \mathrm{g})$, cloxacillin $(5 \mu \mathrm{g})$, and augmentin $(30 \mu \mathrm{g})$ were antibiotics as described in CLSI guideline [26].

2.5. Screening for Multidrug-Resistant Bacteria. Multidrug-resistant bacteria were isolated from wounds. All bacterial cultures were first grown on $5 \% \mathrm{BA}$ plates at $37^{\circ} \mathrm{C}$ for 18-24 hrs before inoculation onto the MHA. Few colonies (3-5) of similar morphology of the respective bacteria were transferred with a sterile inoculating loop to a liquid medium until adequate growth of turbidity with McFarland in 0.5. Then, the bacterial suspension was streaked on MHA plates using a sterile swab in such a way as to ensure thorough coverage of the plates and a uniform thick lawn of growth following incubation. The susceptibilities of clinical isolates were tested by using the MHA containing a range of antimicrobial agents. Dilutions of overnight broth cultures were inoculated onto antibiotic-containing plates to yield final inoculums of approximately $10^{6} \mathrm{CFU}$ per spot for S. aureus, E. faecalis, K. pneumoniae, E. coli, and $P$. aeruginosa according to disk diffusion methods as recommended by CLSI guidelines $[3,26]$.

2.5.1. Screening for Gram-Negative Bacteria. Selected multidrug-resistant Gram-negative bacteria such as ESBL producing E. coli and P. aeruginosa were detected by double disk synergy test (DDST) as described by Jarlier [27]. Mueller Hinton Agar was inoculated with standardized inoculum using sterile cotton swab. Augmentin $(20 \mu \mathrm{g}$ amoxicillin and $10 \mu \mathrm{g}$ of clavulanic acid, AMC) disk was placed in the center of the plate and test disks of cephalosporins (ceftazidime $30 \mu \mathrm{g}$, ceftriaxone $30 \mu \mathrm{g}$, and cefotaxime $30 \mu \mathrm{g}$ ) and aztreonam $30 \mu \mathrm{g}$ disks were placed at $20 \mathrm{~mm}$ distance (center to center) from the amoxicillin-clavulanic acid disk before incubation. The plate was incubated overnight at $35^{\circ} \mathrm{C}$. Enhancement of the zone of inhibition of any one of the four drug disks toward amoxicillin-clavulanic acid suggested the presence of extended-spectrum beta-lactamases. $K$. pneumoniae was screened for its resistance to more than two different classes of antibiotics following disk diffusion method as CLSI guidelines [3, 26, 28].

2.5.2. Screening for Drug Resistant Gram-Positive Bacteria. In this study, cefoxitin was used as the marker of mecA/ mecC mediated by methicillin resistant $S$. aureus and vancomycin (VRE) drug of choice for disk diffusion method as recommended by CLSI guidelines. On the other hand, the concentration values (MIC and $\mathrm{MBC}$ ) and fractional inhibitory concentration index were determined by MHB broth microdilution method as described by Eshetu $[3,26,29,30]$.

2.6. Statistical Analysis. Statistical data were reading values of inhibition zones and concentration values (MIC and MBC) analyzed using SPSS, version 21. Each experiment value is expressed as mean \pm S.D. Statistical significance was determined by student t-test.

Significance was determined by student's $t$-test. Values with $p<0.05$ were considered significant.

\section{Results}

3.1. Antibacterial Effect of Modern Antibiotics. Majority of bacteria isolated from wounds were resistant to two or more antibiotics. In this regard, methicillin resistant $S$. aureus (MRSA) and ESBL producing $P$. aeruginosa were major wound colonizing bacteria. From Gram-positive bacteria, MRSA and VRE were resisted for amikacin, cefoxitin, amoxicine, ampicillin, and cefotaxime (Table 1). On the other hand, Gram-negative bacteria ESBL producing E. coli and $P$. aeruginos $a$ were isolated from surgical wounds. Those Gram-negative bacteria have been undermining in the case of wound care, whereas these bacteria strains were unpredictably resisted for classical modern third-generation cephalosporins and penicillin classes (Table 1). In such cases, gene or gene products of those bacteria transfer intra- or interbacteria species compromised treatment options and worsen the future of medical care.

3.2. Antibacterial Effect of Essential Oils. This study revealed that testing essential oils had broad bactericidal activities. They inhibited growth of E. faecalis, E. coli, P. aeruginosa, $K$. pneumoniae, and S. aureus with zones of IZ ranging from 12-26 mm (Table 2). Thus, oils demonstrated an antibacterial effect against wound colonization MRSA and ESBL producing Gram-negative bacteria. Their effectiveness varied with the concentration and type of bacterial species. For instance, $R$. abyssinicus inhibited the growth of MRSA $(23 \mathrm{~mm})$ and ESBL producing $E$ coli $(22 \mathrm{~mm})$ and P. aeruginosa $(20 \mathrm{~mm})$ at its $20 \mu \mathrm{l} / \mathrm{disc}$, whereas D. penninervium inhibited the growth of MRSA $(21 \mathrm{~mm})$ and ESBL producing $E$ coli $(18 \mathrm{~mm})$ and $P$. aeruginosa $(22 \mathrm{~mm})$ at its $20 \mu \mathrm{l} /$ disc. These essential oils had an 
TABLE 1: Antibiotics resistant profile of multidrug-resistant bacteria isolated from wounds at KMU, 2020.

\begin{tabular}{|c|c|c|c|c|c|c|c|c|c|c|c|c|c|c|c|}
\hline \multirow{2}{*}{ Modern drugs } & \multicolumn{15}{|c|}{ Drug susceptibility test for multidrug bacteria strains isolated from wound samples } \\
\hline & Ef1 & Ef2 & Ef3 & A1 & A2 & A3 & Ec1 & Ec2 & Ec3 & Ar1 & Ar2 & Ar3 & K1 & $\mathrm{K} 2$ & K3 \\
\hline Ciprofloxacin & $\mathrm{R}$ & $\mathrm{R}$ & I & $\mathrm{R}$ & I & $\mathrm{R}$ & $\mathrm{R}$ & $\mathrm{R}$ & $\mathrm{R}$ & $\mathrm{R}$ & $S$ & I & $\mathrm{R}$ & I & $\mathrm{R}$ \\
\hline Cefoxitin & $\mathrm{R}$ & I & $\mathrm{R}$ & $\mathrm{R}$ & $\mathrm{R}$ & $\mathrm{R}$ & $\mathrm{N}$ & $\mathrm{N}$ & $\mathrm{N}$ & $\mathrm{N}$ & $\mathrm{N}$ & $\mathrm{N}$ & $\mathrm{N}$ & $\mathrm{N}$ & $\mathrm{N}$ \\
\hline Cefuroxime & S & $\mathrm{R}$ & I & $\mathrm{N}$ & $\mathrm{N}$ & $\mathrm{N}$ & $\mathrm{R}$ & $\mathrm{R}$ & $\mathrm{R}$ & $\mathrm{R}$ & $\mathrm{R}$ & $\mathrm{R}$ & $\mathrm{I}$ & $\mathrm{R}$ & I \\
\hline Ceftriaxone & $S$ & I & $\mathrm{R}$ & $\mathrm{N}$ & $\mathrm{N}$ & $\mathrm{N}$ & I & $\mathrm{R}$ & $\mathrm{R}$ & $\mathrm{R}$ & $\mathrm{R}$ & $\mathrm{R}$ & $\mathrm{R}$ & $\mathrm{R}$ & $\mathrm{R}$ \\
\hline Ceftazidime & $\mathrm{N}$ & $\mathrm{N}$ & $\mathrm{N}$ & $\mathrm{N}$ & $\mathrm{N}$ & $\mathrm{N}$ & $\mathrm{R}$ & I & $\mathrm{R}$ & $\mathrm{R}$ & $\mathrm{R}$ & $\mathrm{R}$ & $\mathrm{R}$ & $S$ & I \\
\hline Chloramphenicol & $\mathrm{R}$ & $\mathrm{R}$ & $\mathrm{R}$ & $S$ & $\mathrm{R}$ & I & $\mathrm{R}$ & $\mathrm{R}$ & $S$ & $\mathrm{R}$ & $\mathrm{R}$ & $\mathrm{R}$ & $S$ & $\mathrm{R}$ & $\mathrm{R}$ \\
\hline Ampicillin & I & S & S & $\mathrm{R}$ & $\mathrm{R}$ & $\mathrm{R}$ & $\mathrm{S}$ & $\mathrm{R}$ & $\mathrm{R}$ & $\mathrm{S}$ & $\mathrm{R}$ & $\mathrm{R}$ & $\mathrm{R}$ & $\mathrm{R}$ & $\mathrm{R}$ \\
\hline Augmentin & $\mathrm{R}$ & I & $S$ & $\mathrm{R}$ & $\mathrm{I}$ & S & $\mathrm{R}$ & $\mathrm{R}$ & I & $\mathrm{R}$ & $S$ & $\mathrm{R}$ & $\mathrm{R}$ & $\mathrm{I}$ & $S$ \\
\hline Amikacin & $\mathrm{R}$ & $\mathrm{R}$ & $\mathrm{R}$ & $\mathrm{R}$ & $\mathrm{R}$ & $\mathrm{R}$ & $\mathrm{N}$ & $\mathrm{N}$ & S & $\mathrm{R}$ & $\mathrm{R}$ & $\mathrm{R}$ & $\mathrm{I}$ & $\mathrm{R}$ & S \\
\hline Erythromycin & $\mathrm{R}$ & I & $\mathrm{R}$ & $\mathrm{R}$ & $\mathrm{R}$ & $\mathrm{I}$ & $\mathrm{R}$ & I & $\mathrm{R}$ & $\mathrm{R}$ & $\mathrm{R}$ & $\mathrm{R}$ & S & $\mathrm{S}$ & $\mathrm{R}$ \\
\hline Tetracycline & $\mathrm{R}$ & S & I & $\mathrm{R}$ & $\mathrm{R}$ & $\mathrm{R}$ & I & I & $\mathrm{R}$ & $\mathrm{R}$ & $\mathrm{R}$ & $\mathrm{R}$ & S & $\mathrm{R}$ & I \\
\hline Penicillin & $\mathrm{R}$ & I & $\mathrm{R}$ & $\mathrm{R}$ & $\mathrm{R}$ & $\mathrm{R}$ & $\mathrm{R}$ & $\mathrm{R}$ & $\mathrm{R}$ & $\mathrm{R}$ & $\mathrm{R}$ & $\mathrm{R}$ & $\mathrm{R}$ & I & $\mathrm{R}$ \\
\hline Vancomycin & $\mathrm{R}$ & $\mathrm{R}$ & $\mathrm{R}$ & I & $\mathrm{R}$ & S & $\mathrm{N}$ & $\mathrm{N}$ & $\mathrm{N}$ & $\mathrm{N}$ & $\mathrm{N}$ & $\mathrm{N}$ & $\mathrm{N}$ & $\mathrm{N}$ & $\mathrm{N}$ \\
\hline Gentamycin & $\mathrm{R}$ & I & S & $\mathrm{R}$ & $\mathrm{R}$ & $\mathrm{R}$ & $S$ & $\mathrm{R}$ & $\mathrm{R}$ & $\mathrm{S}$ & $\mathrm{R}$ & $\mathrm{I}$ & S & $\mathrm{R}$ & $\mathrm{R}$ \\
\hline
\end{tabular}

$\mathrm{A}=$ methicillin resistant $S$. aureus; $\mathrm{Ar}=\mathrm{ESBL}$ producing $P$. aeruginosa; $\mathrm{Ec}=\mathrm{ESBL}$ producing $E$. coli; $\mathrm{N}=$ not done; $\mathrm{Ef}=\mathrm{Vancomycin}$ resistant $E$. faecalis; $\mathrm{K}=$ multidrug-resistant $K$. pneumoniae; $\mathrm{I}=$ intermediate $(17-20 \mathrm{~mm} \mathrm{IZ);} \mathrm{R}=$ resistance ( $\leq 16 \mathrm{~mm} \mathrm{IZ);} \mathrm{S}=$ sensitive $(\geq 21 \mathrm{~mm}$ IZ) according to CLSI guidelines [25].

TABLE 2: Inhibition zone ( $\mathrm{mm}$ ) of essential oils against MDR bacteria isolated from wounds at KMU, 2020.

\begin{tabular}{|c|c|c|c|c|c|c|c|c|c|c|c|}
\hline \multirow{4}{*}{ Essential oils, $50 \mu \mathrm{l} / \mathrm{ml}$} & \multirow{4}{*}{$\mu \mathrm{l} / \mathrm{disc}$} & \multicolumn{10}{|c|}{ Inhibition zone in diameter $(\mathrm{mm})$, pathogen bacteria isolated from wounds } \\
\hline & & \multicolumn{4}{|c|}{ Gram-positive bacteria } & \multicolumn{6}{|c|}{ Gram-negative bacteria } \\
\hline & & \multicolumn{2}{|c|}{ S. aureus } & \multicolumn{2}{|c|}{ E. faecalis } & \multicolumn{2}{|c|}{ E. coli } & \multicolumn{2}{|c|}{ K. pneumoniae } & \multicolumn{2}{|c|}{ P. aeruginosa } \\
\hline & & ATCC & MRSA & ATCC & VRE & ATCC & ESBL & ATCC & MDR & ATCC & ESBL \\
\hline \multirow{3}{*}{ R. abyssinicus } & 20 & $23 \pm 0.8$ & $23 \pm 3.0^{*}$ & $25 \pm 1.3$ & $25 \pm 1.5^{\# \#}$ & $21 \pm 0.7$ & $22 \pm 0.2 \mathbb{đ}$ & $25 \pm 0.5$ & $25 \pm 0.9^{\$}$ & $21 \pm 0.8$ & 20.9 \\
\hline & 10 & $17 \pm 0.4$ & $18 \pm .0 .1$ & $20 \pm 0.9$ & $19 \pm 1.0$ & $11 \pm 0.3$ & $12 \pm 1.4$ & $17 \pm 1.7$ & $17 \pm 0.1$ & $14 \pm 1.2$ & $14 \pm 2.1$ \\
\hline & 5 & $10 \pm 1.7$ & $9 \pm .0 .9$ & $14 \pm 1.0$ & $13 \pm 1.5$ & NI & NI & $11 \pm 0.6$ & $10 \pm 1.1$ & $8 \pm 2.0$ & $9 \pm 0.1$ \\
\hline \multirow{3}{*}{ D. penninervium } & 20 & $20 \pm 2.0$ & $21 \pm 0.5^{*}$ & $22 \pm 0.1$ & $22 \pm 1.0^{\#}$ & $19 \pm 0.8$ & $18 \pm 0.1$ & $26 \pm 0.7$ & $26 \pm 0.4^{\$}$ & $21 \pm 0.9$ & $22 \pm 0.3^{\ddagger}$ \\
\hline & 10 & $13 \pm 0.1$ & $14 \pm .0 .3$ & $17 \pm 0.4$ & $16 \pm 0.7$ & $14 \pm 0.7$ & $15 \pm 1.0$ & $18 \pm 0.1$ & $17 \pm 0.5$ & $17 \pm 0.6$ & $17 \pm 0.7$ \\
\hline & 5 & $7 \pm 0.7$ & $8 \pm .0 .9$ & $11 \pm 0.1$ & $9 \pm 1.3$ & $9 \pm 1.6$ & $8 \pm 1.0$ & $12 \pm 1.9$ & $9 \pm 0.1$ & NI & NI \\
\hline \multirow{3}{*}{ C. pustulatus } & 20 & $19 \pm 0.1$ & $19 \pm 0.2$ & $20 \pm 0.1$ & $21 \pm 0.1^{\#}$ & $12 \pm 0.7$ & $12 \pm 1.1$ & $22 \pm 0.3$ & $22 \pm 0.4$ & $19 \pm 0.3$ & $19 \pm 0.4$ \\
\hline & 10 & $10 \pm 0.5$ & $9 \pm 0.2$ & $12 \pm 0.6$ & $14 \pm 0.6$ & NI & NI & $13 \pm 0.4$ & $13 \pm 0.9$ & $8 \pm 0.4$ & $11 \pm 0.9$ \\
\hline & 5 & NI & $\mathrm{NI}$ & $9 \pm 0.6$ & $8 \pm 0.3$ & $\mathrm{NI}$ & $\mathrm{NI}$ & $7 \pm 0.6$ & $10 \pm 0.6$ & $\mathrm{NI}$ & NI \\
\hline \multirow{2}{*}{\multicolumn{2}{|c|}{$\begin{array}{l}\text { Positive control } \\
\text { Negative control (5\% DMSO) }\end{array}$}} & $S$ & $\mathrm{R}$ & $S$ & $\mathrm{R}$ & $S$ & $\mathrm{R}$ & $S$ & $\mathrm{R}$ & $S$ & $\mathrm{R}$ \\
\hline & & $\mathrm{NI}$ & $\mathrm{NI}$ & $\mathrm{NI}$ & $\mathrm{NI}$ & NI & $\mathrm{NI}$ & $\mathrm{NI}$ & $\mathrm{NI}$ & $\mathrm{NI}$ & NI \\
\hline
\end{tabular}

Mean \pm SD,$C=$ ciprofloxacin; $\mathrm{NI}=$ no inhibition zone; $\mathrm{NT}=$ not tested; ATCC $=$ susceptible $>21 \mathrm{~mm} \mathrm{IZ}$, resistant $<16 \mathrm{~mm} \mathrm{IZ}$, where $P<0.05$ when compared to cefoxitin-treated MRSA, while ${ }^{\#} P<0.05$ and ${ }^{\# \#} P<0.01$ when compared to modern drug treated E. faecalis and ${ }^{\$} P<0.05$ when compared to K. pneumoniae; ${ }^{¥} P<0.05$ when compared to modern drug treated $P$. aeruginosa, and ${ }^{\Phi} P<0.05$ when compared to modern drug treated E. coli.

inhibitory effect on both reference strains and MDR bacteria with IZ ranging from $9-26 \mathrm{~mm}$ in diameter and MIC and $\mathrm{MBC}$ values ranging from $2-32 \mu \mathrm{l} / \mathrm{ml}$. The EOs of $R$. abyssinicus, C. pustulatus, and D. penninervium showed IZ in diameter values $23 \mathrm{~mm}, 19 \mathrm{~mm}$, and $21 \mathrm{~mm}$ on the MRSA, respectively. Vancomycin resistant E. faecalis were highly susceptible for tested EOs as compared to other species.

The essential oil of $R$. abyssinicus was powerful antibacterial active against Gram-positive and Gram-negative bacteria in general and VRE and K. pneumoniae in particular with $25 \mathrm{~mm} \mathrm{IZ} \mathrm{at} 20 \mu \mathrm{l} /$ disc. Likewise, $R$. abyssinicus, C. pustulatus, and D. penninervium showed IZ $20 \mathrm{~mm}$, $19 \mathrm{~mm}$, and $22 \mathrm{~mm}$, respectively, on ESBL producing $P$. aeruginosa that were second common bacteria isolates from wounds. Those EOs had MIC and MBC values ranging from $4-32 \mu \mathrm{l} / \mathrm{ml}$. Overall, tested EOs can supersede wound colonization and biofilm forming bacteria (Table 3 ).

3.3. Combined Antibacterial Effect of Essential Oils. This study revealed that combination of essential oils $1: 1$ ratio was more strong antibacterial effect than individual essential oil alone and even equivalent to modern drug of choice. The combined EOs inhibited the growth of MRSA and ESBL producing $E$ coli and $P$. aeruginosa and their reference strains at $20 \mu \mathrm{l} / \mathrm{disc}$ (Table 3 ), of which the combined EO obtained from $R$. abyssinicus and D. penninervium (1:1 ratio) exhibited the strongest antibacterial activities. It had IZ in diameters $32 \mathrm{~mm}, 34 \mathrm{~mm}$, and $19 \mathrm{~mm}$ on MRSA, VRE, and ESBL producing 
TABLE 3: Inhibition zone (mm) of combined essential oils against MDR bacteria isolated from wounds at KMU, 2020.

\begin{tabular}{|c|c|c|c|c|c|c|c|c|c|c|c|}
\hline \multirow{4}{*}{ Essential oils, $50 \mu \mathrm{l} / \mathrm{ml}$} & \multirow{4}{*}{$\mu \mathrm{l} / \mathrm{disc}$} & \multicolumn{10}{|c|}{ Inhibition zone in diameter $(\mathrm{mm})$, pathogen bacteria isolated from wounds } \\
\hline & & \multicolumn{4}{|c|}{ Gram-positive bacteria } & \multicolumn{6}{|c|}{ Gram-negative bacteria } \\
\hline & & \multicolumn{2}{|c|}{ S. aureus } & \multicolumn{2}{|c|}{ E. faecalis } & \multicolumn{2}{|c|}{ E. coli } & \multicolumn{2}{|c|}{ K. pneumoniae } & \multicolumn{2}{|c|}{ P. aeruginosa } \\
\hline & & ATCC & MRSA & ATCC & VRE & ATCC & ESBL & ATCC & MDR & ATCC & ESBL \\
\hline $\begin{array}{l}\text { R. abyssinicus and } \\
\text { C. pustulatus }\end{array}$ & $\begin{array}{c}20 \\
10 \\
5\end{array}$ & $\begin{array}{l}29 \pm 2.1 \\
19 \pm 0.5 \\
13 \pm 0.7\end{array}$ & $\begin{array}{c}29 \pm 1.3^{*} \\
16 \pm .2 .1 \\
14 \pm .0 .5\end{array}$ & $\begin{array}{l}31 \pm 1.3 \\
20 \pm 0.7 \\
10 \pm 0.6\end{array}$ & $\begin{array}{c}31 \pm 0.6^{\#} \\
21 \pm 1.4 \\
11 \pm 0.6\end{array}$ & $\begin{array}{l}23 \pm 2.7 \\
17 \pm 0.4 \\
10 \pm 0.3\end{array}$ & $\begin{array}{c}22 \pm 1.7 \\
18 \pm 1.0 \\
9 \pm 1.4\end{array}$ & & $\begin{array}{l}33 \pm 0.1 \\
23 \pm 1.9 \\
16 \pm 2.4\end{array}$ & $\begin{array}{l}26 \pm 0.7 \\
18 \pm 1.1 \\
11 \pm 0.8\end{array}$ & $\begin{array}{c}26 \pm 0.9^{¥} \\
18 \pm 1.4 \\
8 \pm 0.4\end{array}$ \\
\hline $\begin{array}{l}\text { C. pustulatus and } \\
\text { D. penninervium }\end{array}$ & $\begin{array}{c}20 \\
10 \\
5 \\
\end{array}$ & $\begin{array}{l}27 \pm 1.0 \\
19 \pm 0.9 \\
12 \pm 0.3\end{array}$ & $\begin{array}{c}25 \pm 0.5^{*} \\
17 \pm .0 .1 \\
11 \pm .0 .8 \\
\end{array}$ & $\begin{array}{l}33 \pm 0.1 \\
27 \pm 1.5 \\
17 \pm 0.4\end{array}$ & $\begin{array}{c}32 \pm 1.9^{\# \#} \\
27 \pm 0.1 \\
13 \pm 0.8\end{array}$ & $\begin{array}{c}27 \pm 1.4 \\
19 \pm 0.4 \\
9 \pm 0.3\end{array}$ & & & & $\begin{array}{l}28 \pm 0.7 \\
18 \pm 2.4 \\
12 \pm 0.1\end{array}$ & \\
\hline $\begin{array}{l}\text { D. penninervium and } \\
\text { R. abyssinicus }\end{array}$ & $\begin{array}{c}20 \\
10 \\
5\end{array}$ & $\begin{array}{l}32 \pm 1.1 \\
26 \pm 2.5 \\
13 \pm 2.1\end{array}$ & $\begin{array}{c}32 \pm 1.2^{* *} \\
26 \pm 0.3 \\
14 \pm .0 .8\end{array}$ & $\begin{array}{l}34 \pm 0.4 \\
25 \pm 0.6 \\
16 \pm 0.4\end{array}$ & $\begin{array}{c}31 \pm 2.1^{\# \#} \\
24 \pm 0.9 \\
17 \pm 0.7\end{array}$ & $\begin{array}{c}23 \pm 1.3 \\
17 \pm 1.7 \\
9 \pm 1.7\end{array}$ & $\begin{array}{c}22 \pm 0.4 \\
16 \pm 0.8 \\
8 \pm 0.5\end{array}$ & $\begin{array}{l}29 \pm 0.3 \\
20 \pm 0.6 \\
15 \pm 0.2\end{array}$ & $\begin{array}{l}29 \pm 0.4 \\
20 \pm 0.4 \\
16 \pm 0.7\end{array}$ & $\begin{array}{c}21 \pm 0.3 \\
13 \pm 0.4 \\
8 \pm 1.8\end{array}$ & $\begin{array}{c}19 \pm 0.4 \\
14 \pm 0.9 \\
8 \pm 1.3\end{array}$ \\
\hline $\begin{array}{l}\text { Combination of } \\
\text { three }(1: 1: 1 \text { ratio })\end{array}$ & $\begin{array}{c}20 \\
10 \\
5\end{array}$ & $\begin{array}{l}34 \pm 0.3 \\
26 \pm 0.1 \\
17 \pm 1.2\end{array}$ & $\begin{array}{c}33 \pm .1 .5^{* *} \\
26 \pm .1 .1 \\
16 \pm .0 .9\end{array}$ & $\begin{array}{l}37 \pm 0.2 \\
24 \pm 0.8 \\
18 \pm 0.7\end{array}$ & $\begin{array}{c}37 \pm 1.7^{\# \#} \\
25 \pm 0.9 \\
18 \pm 0.8\end{array}$ & $\begin{array}{c}22 \pm 1.1 \\
15 \pm 1.2 \\
9 \pm 0.7\end{array}$ & $\begin{array}{c}22 \pm 1.6 \\
16 \pm 0.1 \\
9 \pm 0.4\end{array}$ & $\begin{array}{l}35 \pm 1.7 \\
25 \pm 2.0 \\
16 \pm 0.3\end{array}$ & $\begin{array}{l}35 \pm 0.2 \\
25 \pm 1.4 \\
17 \pm 0.9\end{array}$ & $\begin{array}{l}26 \pm 0.8 \\
17 \pm 0.5 \\
12 \pm 0.1\end{array}$ & $\begin{array}{c}26 \pm 0.4^{¥} \\
17 \pm 0.2 \\
11 \pm 1.9\end{array}$ \\
\hline $\begin{array}{l}\text { Positive control } \\
\text { Negative control }\end{array}$ & & $\begin{array}{c}\mathrm{S} \\
\mathrm{NI}\end{array}$ & $\begin{array}{c}\mathrm{R} \\
\mathrm{NI}\end{array}$ & $\begin{array}{c}\mathrm{S} \\
\mathrm{NI}\end{array}$ & $\begin{array}{c}\mathrm{R} \\
\mathrm{NI}\end{array}$ & $\begin{array}{c}\mathrm{S} \\
\mathrm{NI}\end{array}$ & $\begin{array}{c}\mathrm{R} \\
\mathrm{NI} \\
\end{array}$ & $\begin{array}{c}\text { S } \\
\text { NI }\end{array}$ & $\begin{array}{c}\mathrm{R} \\
\mathrm{NI}\end{array}$ & $\begin{array}{c}\mathrm{S} \\
\mathrm{NI} \\
\end{array}$ & $\begin{array}{c}\mathrm{R} \\
\mathrm{NI} \\
\end{array}$ \\
\hline
\end{tabular}

Mean $\pm \mathrm{SD}$, whereas $\mathrm{C}=$ ciprofloxacin; $\mathrm{NI}=$ no inhibition zone; $\mathrm{NT}=$ not tested; $\mathrm{ATCC}=$ susceptible $>21 \mathrm{~mm} \mathrm{IZ,} \mathrm{resistant}<16 \mathrm{~mm} \mathrm{IZ,} \mathrm{where}{ }^{*} P<0.05$ and ${ }^{* *} P<0.01$ when compared to cefoxitin treated MRSA, while ${ }^{\#} P<0.05$ and ${ }^{\# \#} P<0.01$ when compared to modern drug treated $E$. faecalis; ${ }^{\circledR} P<0.05$ and ${ }^{\$ \$} P<0.01$ when compared to $K$. pneumoniae and ${ }^{¥} P<0.05$ when compared to modern drug treated $P$. aeruginosa, and ${ }^{\Phi} P<0.05$ when compared to modern drug treated E. coli.

TABle 4: MIC and MBC values of essential oils against human pathogenic bacteria in agar dilution method at KMU, 2020.

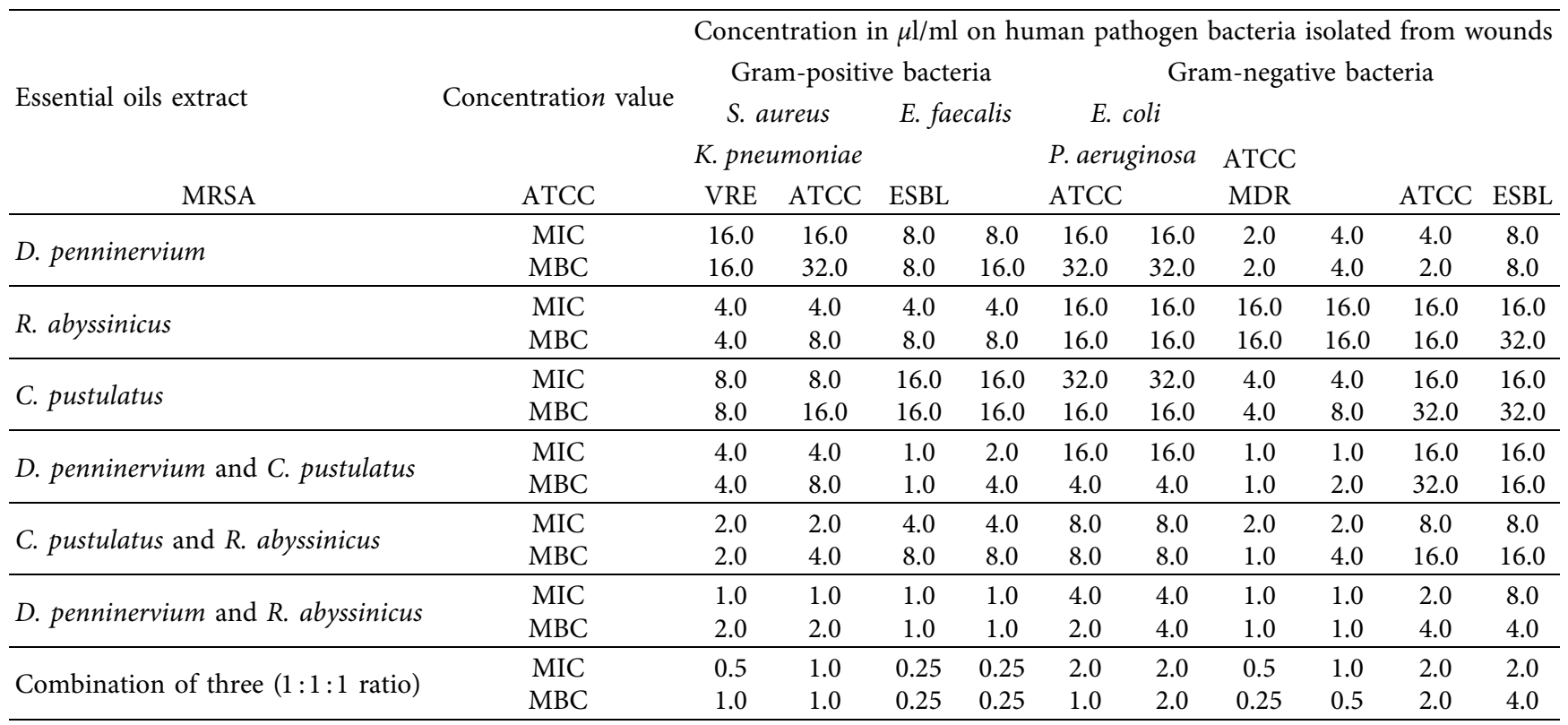

Modern drug

$\mathrm{ATCC}=$ reference strain for each species; $\mathrm{MDR}=$ multidrug-resistant strains; $\mathrm{MBC}=$ minimal bactericidal concentration; $\mathrm{MIC}=$ minimal inhibitory concentration; $\mathrm{NT}=$ not tested; $C=5 \mu \mathrm{g}$ ciprofloxacin +growth has been seen.

P. aeruginosa and E. coli at $20 \mu \mathrm{l} / \mathrm{disc}$, respectively. This mixture had the lowest MIC and MBC values as compared to the other mixture. The MIC value for VRE was $1.0 \mu \mathrm{l} / \mathrm{ml}$ and MRSA $(2.0 \mu \mathrm{l} / \mathrm{ml})$. The MBC values were ranging from $1.0-8.0 \mu \mathrm{l} / \mathrm{ml}$ for ESBL producing $P$. aeruginosa (Table 4). This combined oil showed powerful bactericidal effect as compared to modern antibiotics against tested reference and drug-resistant bacteria species.
The combination of C. pustulatus and R. abyssinicus essential oils had $29 \mathrm{~mm}$ IZ in diameter against both MSSA and MRSA. It showed an effective antibacterial effect on $K$. pneumoniae and E. coli. It had MIC values ranging from $2.0-8.0 \mu \mathrm{l} / \mathrm{ml}$ for tested Gram-negative bacteria and $2.0 \mu \mathrm{l} /$ $\mathrm{ml}$ against MRSA. It had MBC value $4.0 \mu \mathrm{l} / \mathrm{ml}$ for K. pneumonia and MRSA and $16 \mu \mathrm{l} / \mathrm{ml}$ against ESBL producing $P$. aeruginosa. Similarly, the combined effect of EOs 
TABLE 5: The mean fractional inhibitory concentration index for MDR bacteria at KMU, 2020.

\begin{tabular}{|c|c|c|c|c|c|}
\hline \multirow{3}{*}{ Mixture of oil in $1: 1$ ratio } & \multicolumn{2}{|c|}{ Gram positive } & \multicolumn{3}{|c|}{ Gram negative } \\
\hline & \multirow{2}{*}{ MRSA } & \multirow{2}{*}{ VRE } & \multirow{2}{*}{ MDR K. pneumoniae } & \multicolumn{2}{|c|}{ ESBL producing bacteria } \\
\hline & & & & E. coli & P. aeruginosa \\
\hline $\begin{array}{l}\text { D. penninervium } \\
\text { C. pustulatus }\end{array}$ & $0.75^{*}$ & $1.25^{\#}$ & $0.625^{*}$ & $0.75^{*}$ & $1.0^{*}$ \\
\hline $\begin{array}{l}\text { C. pustulatus } \\
\text { R. abyssinicus }\end{array}$ & $0.75^{*}$ & $0.375^{¥}$ & $0.5^{*}$ & $1.5^{\#}$ & $3.0^{\#}$ \\
\hline $\begin{array}{l}\text { D. penninervium } \\
\text { R. abyssinicus }\end{array}$ & $0.3125^{¥ ¥}$ & $0.375^{¥}$ & $0.3125^{¥}$ & $0.5^{*}$ & $0.5^{*}$ \\
\hline
\end{tabular}

The values represent mean fractional inhibitory concentration index $(n=6)$, where ${ }^{¥} X=$ synergy interaction, ${ }^{¥} X=$ partial synergy, ${ }^{*} X=$ indifference interaction, and ${ }^{\#} X=$ antagonistic interaction of essential oil.

obtained from $R$. abyssinicus and D. penninervium had $29 \mathrm{~mm}$ and $22 \mathrm{~mm} \mathrm{IZ} \mathrm{in} \mathrm{diameter} \mathrm{at} 20 \mu \mathrm{l} /$ disc against K. pneumoniae and E. coli, respectively (Table 3 ). It had also $32 \mathrm{~mm}$ IZ in diameter on MSSA and MRSA. Interestingly, combined effect of three EOs within $1: 1: 1$ had $34 \mathrm{~mm}$, $37 \mathrm{~mm}$, and $26 \mathrm{~mm}$ on MRSA, VRE, and ESBL producing $P$. aeruginosa, respectively. This combination had MIC and $\mathrm{MBC}$ ranging from $0.25-4.0 \mu \mathrm{l} / \mathrm{ml}$ for tested bacteria. Overall, the combination of each EO had more potent antibacterial effect on MRSA and $K$. pneumoniae, $P$. aeruginosa, and E. coli as compared to currently available and affordable antibiotics (Table 1).

The fractional inhibitory concentration index (FIC index) revealed that the combined essential oils from $R$. abyssinicus and D. penninervium in 1:1 ratio had synergistic effect on MRSA, VRE, and K. pneumoniae. It had also adaptive effect on ESBL producing $E$. coli and $P$. aeruginosa. On the other hand, mixture of $R$. abyssinicus and $C$. pustulatus had synergistic effect on VRE and additive on MRSA and K. pneumoniae. Conversely, it had antagonistic effect on ESBL producing E. coli and P. aeruginosa. The mixture of essential oils from C. pustulatus and $D$. penninervium showed additive effect on MRSA and K. pneumoniae. Its FIC index values were 075 and 0.625 for MRSA and K. pneumoniae, respectively. Overall, MRSA was most susceptible for combined essential oil. In contrary to this, $P$. aeruginosa resisted bacteria as compared to other bacteria for combined oil (Table 5).

\section{Discussion}

This study revealed that MRSA, VRE, and ESBL producing $E$. coli and $P$. aeruginosa were human pathogen MDR bacteria isolated from wound samples. Those strains were compromised for all or the majority of antibiotics used in low- and middle-income countries (Table 1). Similarly, many studies showed an alarming increase in multidrugresistant bacteria on a global scale [3, 21-23]. Their resistance genes or gene products transfer into intra-and interspecies by transformation, transduction, and conjugation in very dynamic and unpredictable phenomena $[16,27]$. On the other hand, almost all hospitals in developing countries have no drug susceptibility facilities to diagnose patients and administrated clinically. In this way, infected wounds with multidrug-resistant bacteria have limited treatment options and escalating mortality, morbidity rates, and treatment costs $[13,15]$. These are ways that caused them to infect and spreading at the community level. As a result, they imposed a potentially large health and socioeconomic burden on societies and worries about the future provision of medicalsurgical and other healthcare services [10]. In such cases, traditional healers use their ingenious knowledge such as medicinal plants to treat many ailments including chronic wound ulcers [8-10]. Since pastoralist communities are deprived of modern medical care and depend on the medicinal plants, they are resourceful for generating knowledge to tackle multidrug-resistant microbes in general and wound colonizing species in particular [3]. Those communities extracted essential oils from medicinal plants and used as a topical ointment for wound care in rural pastoralist communities [12, 19].

These study findings showed that almost all tested essential oils had promising antibacterial activity on the multidrug-resistant pathogenic bacteria colonizing and triggering wound infection, of which MRSA was more susceptible to tested essential oils in combination and/or alone. Likewise, VRE was also inhibited by most tested essential oils at $20 \mu \mathrm{l} / \mathrm{disc}$. These findings substantiate earlier studies that the therapeutic agents derived from essential oils can devastate the cell wall of bacteria and later kill them. Many compounds such as leptospermone, tricyclene, flavesone, myrcene, carvacrol, p-cymene, and eugenol are able to activate humoral and cellular immunity $[14,15]$. Some of those compounds reduce proinflammatory cytokine and TNF resulting in improvement of wound healing $[5,8,23,29]$. Another study demonstrated that carvacrol of essential oil has bactericidal property [6]. It inhibits protein and nucleic acid synthesis of Gram-positive bacteria, more specifically MRSA and VRE $[7,11]$. Therefore, essential oils are candidate to treat chronic skin ulcer $[9,10,12]$.

With other respects of Gram-negative bacteria, essential oils inhibited the growth of ESBL producing $P$. aeruginosa and E. coli and K. pneumoniae isolate of an infected wound. Bactericidal activity of essential oils was remarkable and pronounced inhibitory effect on references and drug-resistant bacteria. This insight into the application of essential oils on wound can improve healing. On the other hand, doses, types, and bacteria species determine the effectiveness of oils. These findings agreeing with essential oils can reduce biofilm formation and multiplication and inhibit 
inflammatory edema formation and leucocyte chemotaxis around the wound $[16,17,19]$. There were several determinants of acquired resistance to conventional drugs. In line with this, $P$. aeruginosa and E. coli developed resistance genes to many available and affordable antibiotics [20,25]. These are associated with spontaneous mutations that interfere with drug-target binding and compromise prodrug activation or cause over expression of the target [24, 31]. Other studies showed that there were intrinsic genes, acquired antibiotic resistance (expressed new trait), and caused the genetic change. The genetic changes can confer resistance to antibiotics by altering the target site of the drug, enzymatical inactivation of the drug, and preventing the drug from accessing the target sites. Those factors compromised all or third-generation cephalosporins and penicillin classes [15]. Overall, those factors contributed to resisted amoxicillin, tetracycline, piperacillin, ofloxacin, and oxacillin $[16,18,20]$.

This study revealed that combined effect of essential oils was more effective and efficient antibacterial effect than used alone and even equal to modern drug of choice. These synergistic combinations signify to exploited novel compounds from essential oils. This is also the enhanced bactericidal effect of the compounds. In such case, essential oils are suitable candidates to develop topical ointments for wound care and beyond [9]. Another study demonstrated that EOs caused sequential inhibition of biochemical pathway, inhibit protein synthesis, and disintegrated the outer cell membrane $[18,28]$. Overall, essential oils have compounds that used to produce affordable and safe antibiotics and potentially activate the immune system for fast wound healing. The EOs have therapeutic compounds that exert beneficial pharmacological potential on wound care to overcome the problem from multidrug-resistant bacteria.

\section{Conclusions}

This study revealed that testing combined effect of essential oils had broad bactericidal activities, of which they demonstrated more strong antibacterial effect against common wound colonization of MRSA and ESBL producing $P$. aeruginosa. Moreover, majority of combined oil had synergistic and additive effect which provided information to search a novel compound in combination of essential oils or alone. Hence, identifying and structural elucidation of compounds with immunomodulatory and bactericidal effect from essential oils help pharmaceutical companies and researchers to develop less toxic, safe, and affordable drugs or modulators or precursors for the synthesis of new antimicrobial drugs and/or ointment to treat wounds.

\section{Abbreviations}

ATC: American Type Culture Collection used as reference strains for respective bacteria

BA: Blood agar

CLSI: Clinical Laboratory Standard Institute

DDST: Double disk synergy test
EOs: Essential oils obtained from the respective medicinal plant by steam distillation

ESBL: Extended-spectrum beta-lactamase

FIC: Fractional inhibitory concentration index

MBC: Minimum bactericidal concentration

MDR: Multidrug resistant

MHA: Mueller Hinton Agar

MHB: Mueller Hinton Broth

MRSA: Methicillin resistant Staphylococcus aureus

MBC: Minimal bactericidal concentration

MIC: Minimal inhibitory concentration

VRE: Vancomycin resistant E. faecalis.

\section{Data Availability}

All data and materials of this work are available from the corresponding author on request.

\section{Conflicts of Interest}

The authors have declared that they have no conflicts of interest.

\section{Authors' Contributions}

EG and HU designed, extracted, and performed laboratory works and participated in the plant sample collection and analysis of data. All authors read and approved the final manuscript.

\section{Acknowledgments}

The authors would like to acknowledge KMU for the financial support of this research project. They would like to extend their gratitude to the administration and staff members of KMU, MLT department, for assistance and their laboratory facilities. They also would like to thank genuine taxonomist Dr. Melaku Wondafrash for identifying plant samples at the National Herbarium of AAU. Last, not the least, their special thanks go to Ato Tufa Seboke, W/ro Simegne Terefe, Ato Shafi Mohammed, Ato Kasim Kedir, Mequanint Mitiku, Ato Sultan Mohammed, W/ro Kemeru Waliy, S/r Nigatua Desta, and Ato Deme Birhanu for their respective support during plant collection and unreserved encouragement during this research project. The research has been funded by Kotebe Metropolitan University, Addis Ababa, Ethiopia.

\section{References}

[1] B. Petrovska, "Historical review of medicinal plants' usage," Pharmacognosy Reviews, vol. 6, no. 11, pp. 1-5, 2012.

[2] L. T. Ngo, J. I. Okogun, and W. R. Folk, "21st Century natural product research and drug development and traditional medicines," Natural Product Reports, vol. 30, no. 4, pp. 584-592, 2013.

[3] E. Gadisa, G. Weldearegay, K. Desta et al., "Combined antibacterial effect of essential oils from three most commonly used Ethiopian traditional medicinal plants on multidrug 
resistant bacteria," $B M C$ Complementary and Alternative Medicine, vol. 19, no. 1, p. 24, 2019.

[4] S. Y. Pan, S. F. Zhou, S. H. Gao et al., "New perspectives on how to discover drugs from herbal medicines: cam's outstanding contribution to modern therapeutics," EvidenceBased Complementary and Alternative Medicine, vol. 2013, Article ID 627375, 25 pages, 2013.

[5] G. Zhao, M. L. Usui, S. I. Lippman et al., "Biofilms and inflammation in chronic wounds," Advances in Wound Care, vol. 2, no. 7, pp. 389-399, 2013.

[6] L. Ze-Hua, M. Cai, L. Yuan-Shuai, S. Pei-Long, and L. ShaoLei, "Antibacterial activity and mechanisms of essential oil from Citrus medica L. Var. Sarcodactylis," Molecules, vol. 24, no. 8, p. 1577, 2019.

[7] U. Anand, N. Jacobo-Herrera, A. Altemimi, and N. Lakhssassi, "A comprehensive review on medicinal plants as antimicrobial therapeutics: potential avenues of biocompatible drug discovery," Metabolites, vol. 9, no. 11, p. 258, 2019.

[8] T. V. A. Lordani, C. E. Lara, F. B. P. Ferreira et al., "Therapeutic effects of medicinal plants on cutaneous wound healing in humans: a systematic review," Mediators of Inflammation, vol. 201812 pages, 2018.

[9] M. Olsson, K. Järbrink, U. Divakar et al., "The humanistic and economic burden of chronic wounds: a systematic review," Wound Repair and Regeneration, vol. 27, no. 1, pp. 114-125, 2019.

[10] S. R. Nussbaum, M. J. Carter, C. E. Fife et al., "An economic evaluation of the impact, cost, and medicare policy implications of chronic nonhealing wounds," Value in Health, vol. 21, no. 1, pp. 27-32, 2018.

[11] C. K. Sen, "Human wounds and its burden: an updated compendium of estimates," Advances in Wound Care, vol. 8, no. 2, pp. 39-48, 2019.

[12] C. Lindholm and R. Searle, "Wound management for the 21st century: combining effectiveness and efficiency," International Wound Journal, vol. 13, no. 2, pp. 5-15, 2016.

[13] M. M. Mihai, A. M. Holban, C Giurcăneanu et al., "Identification and phenotypic characterization of the most frequent bacterial etiologies in chronic skin ulcers," Romanian Journal of Morphology and Embryology, vol. 55, no. 4, pp. 1401-1408, 2014.

[14] P. S. Yap, S. H. Lim, C. P. Hu, and B. C. Yiap, "Combination of essential oils and antibiotics reduce antibiotic resistance in plasmid-conferred multidrug resistant bacteria," Phytomedicine, vol. 20, no. 8-9, pp. 710-713, 2013.

[15] W. Shan, Y. Wang, Z. Zhang et al., "Qingre Baidu mixtureinduced effect of AI-2 on S. aureus and P. aeruginosa biofilms in chronic and refractory wounds," Experimental and Therapeutic Medicine, vol. 17, pp. 3343-3350, 2019.

[16] J. Espinoza, A. Urzúa, L. Sanhueza et al., "Essential oil, extracts, and sesquiterpenes obtained from the heartwood of Pilgerodendron uviferum act as potential inhibitors of the S. aureus NorA multidrug efflux pump," Frontiers in Microbiology, vol. 26, no. 10, p. 337, 2019.

[17] A. Petruta, M. Anca, T. Adriana, B. Alexandra, G. Elvira, and C. A. Ana, "Essential oils and their components as modulators of antibiotic activity against gram-negative bacteria," Medicines, vol. 3, no. 3, 19 pages, 2016.

[18] C. D. Morais Oliveira-Tintino, S. R. Tintino, P. W. Limaverde et al., "Inhibition of the essential oil from Chenopodium ambrosioides L. and $\alpha$-terpinene on the NorA efflux-pump of S. aureus," Food Chemistry, vol. 262, pp. 72-77, 2018.
[19] M. D. Falcone and D. Paterson, "Spotlight on ceftazidime/ avibactam: a new option for MDR Gram-negative infections," Journal of Antimicrobial Chemotherapy, vol. 71, no. 10, pp. 2713-2722, 2016.

[20] H. Qaralleh, "Thymol rich thymbra capitata essential oil inhibits quorum sensing, virulence and biofilm formation of beta lactamase producing Pseudomonas aeruginosa," Natural Product Sciences, vol. 25, no. 2, p. 172, 2019.

[21] P. W. Limaverde, F. F. Campina, F. A. B. Da Cunha et al., "Inhibition of the TetK efflux-pump by the essential oil of Chenopodium ambrosioides L. and $\alpha$-terpinene against Staphylococcus aureus IS-58," Food and Chemical Toxicology, vol. 109, no. Pt 2, pp. 957-961, 2017.

[22] Q. Benameur, T. Gervasi, V. Pellizzeri et al., "Antibacterial activity of Thymus vulgaris essential oil alone and in combination with cefotaxime against blaESBL producing multidrug resistant Enterobacteriaceae isolates," Natural Product Research, vol. 33, no. 18, pp. 2647-2654, 2019.

[23] S. Varsha, K. Tushar, B. Rohit, S. Ravi, and K. Vinay, "Inhibiting bacterial drug efflux pumps via phyto-therapeutics to combat threatening antimicrobial resistance," Frontiers in Microbiology, vol. 9, p. 2990, 2018.

[24] S. Y. Song, J. E. Hyun, J.-H. Kang, and C. Y. Hwang, "In vitro antibacterial activity of the manuka essential oil from Leptospermum scoparium combined with Tris-EDTA against Gram-negative bacterial isolates from dogs with otitis externa," Veterinary Dermatology, vol. 31, no. 2, pp. 81-85, 2020.

[25] I. Utchariyakiat, S. Surassmo, M. Jaturanpinyo et al., "Efficacy of cinnamon bark oil and cinnamaldehyde on anti-multidrug resistant $P$. aeruginosa and the synergistic effects in combination with other antimicrobial agents," BMC Complement Alternative Medicine, vol. 158, 2016.

[26] CLSI, Performance for Antimicrobial Susceptibility Testing: Twenty-First Informational Supplement-M 100-S21, CLSI, Wayne, PA, USA, 2019.

[27] V. Jarlier, M.-H. Nicolas, G. Fournier, and A. Philippon, "Extended broad-spectrum -lactamases conferring transferable resistance to newer -lactam agents in Enterobacteriaceae: hospital prevalence and susceptibility patterns," Clinical Infectious Diseases, vol. 10, no. 4, pp. 867-878, 1988.

[28] D. Muntean, M. Licker, E. Alexa et al., "Evaluation of essential oil obtained from Menthaxpiperita L. against multidrug-resistant strains," Infection and Drug Resistance, vol. 12, pp. 2905-2914, 2019.

[29] B. S. Farisa, D. Rubini, S. Rakshitaa et al., "Antivirulent properties of underexplored cinnamomum tamala essential oil and its synergistic effects with DNase against Pseudomonas aeruginosa biofilms-an in vitro study," Frontiers in Microbiology, vol. 8, p. 1144, 2017.

[30] I. Wiegand, K. Hilpert, and R. E. W. Hancock, “Agar and broth dilution methods to determine the minimal inhibitory concentration (MIC) of antimicrobial substances," Nature Protocols, vol. 3, no. 2, pp. 163-175, 2008.

[31] R. Kotoky, M. Bordoloi, A. Yadav et al., "Chemical composition and antibacterial activity of essential oil from fruit of Micromelum integerrimum (Buch.-Ham. ex DC.) Wight \& Arn. ex M. Roem," Natural Product Research, vol. 33, no. 24, pp. 3573-3576, 2019. 